\title{
Avaliação de oficinas de autocuidado aos portadores de periodontite crônica: cuidando dos usuários e cirurgiões-dentistas
}

\author{
The evaluation of self-care workshops to carriers \\ of chronic periodontitis: taking care of users and dentists
}

\author{
Sharmênia de Araújo Soares N uto ${ }^{1}$ \\ Samuel Ilo Fernandes de Amorim ${ }^{2}$ \\ $M$ arilyn Kay Nations ${ }^{3}$ \\ Íris do Céu Clara Costa ${ }^{1}$
}

${ }^{1}$ Programa de

Pós-Graduação em Ciências da Saúde, Centro de

Ciências da Saúde,

UniversidadedeFortaleza.

Av. Washington Soares

1321, Edson Queiroz.

60811-341 Fortaleza CE.

UniversidadedeFortaleza. nuto@unifor.br

${ }^{2}$ Secretaria de Saúde,

Prefeitura M unicipal de

Quixelô.

${ }^{3}$ Centro de Ciências da

Saúde, Universidadede

Fortaleza.
Abstract The objective of this article is to evaluate the workshops of "accepting the patient" for dentists and of self-care for carriers of chronic periodontitis. The patients assisted at the periodontics clinics of graduation and post-graduation Dentistry courses and periodontics specialty students, in Fortaleza, Ceará State, Brazil, took part of educative workshops. A field diary was done in all workshops. The patients of both interventions and control groups answered a structured questionnaire at the beginning and at the end of the treatment. In order to compare the previous and post moments, in both groups, a $\mathrm{M}$ cN emar test was performed, with a probability of $p<0.05$. In the intervention group, there was an increase in knowledge related to the name, the causes and related factors of the disease $(p<0.05)$. In the control group there wasn't a rise in the understanding of the diseases' chronic character, unlike the intervention group. The dentists showed few abilities in dealing with chronic patients. It can be concluded that the educative workshops for carriers of chronic periodontitis increase the knowledge about the illness, its causes and its chronic character, especially by the pedagogical methodology used and the dialogue with the popular culture.

Key words Periodontitis, M otivation, Self care, $\mathrm{H}$ ealth education, Chronic disease
Resumo 0 objetivo desteartigo éavaliar as oficinas de "aceitação ao paciente" para os cirurgiõesdentistas e de autocuidado para os portadores de periodontite crônica. Pacientes em atendimento nas clínicas de periodontia dos cursos de graduação e pós-graduação em odontologia e alunos de especialização em periodontia, em Fortaleza (CE), participaram de oficinas educativas. Os pacientes do grupo de intervenção e controle responderam a um questionário estruturado no início e no final do tratamento. Foi realizado o diário de campo em todas as oficinas. Para a comparação entre os momentos antes e depois, nos dois grupos, foi realizado o teste $\mathrm{M}$ cNemar, com uma probabilidade de $p<0,05$. No grupo de intervenção, houve um incremento do conhecimento em relação ao nome, às causas e aos fatores relacionados à doença $(p<$ $0,05)$. No grupo-controle, não houve aumento na compreensão do caráter crônico, ao contrário do grupo de intervenção. Os cirurgiões-dentistas demonstraram pouca habilidade em lidar com pacientes crônicos. As oficinas educativas aos portadores de periodontite crônica acrescentam conhecimentos sobre a doença, suas causas e seu caráter crônico, principalmente pela metodologia pedagógica utilizada e o diálogo com a cultura popular. Palavras-chave Periodontite, Motivação, Autocuidado, Educação em saúde, D oença crônica 


\section{Introdução}

A educação em saúde tradicional permeia, basicamente, os grandes meios de comunicação de massa e os serviços primários de saúde, enfatizando a estruturação de instrumentos educativos, com vistas a propiciar a interação cultural entre população e profissionais de saúde, buscando nestas práticas ensinar aos pacientes os cuidados com o corpo, a saúde, a prevenção e o tratamento das doenças ${ }^{1}$.

As práticas coercitivas do final do século XIX e início do século $X X$, baseadas na pedagogia da higiene, foram substituídas gradativamente por atividades educativas centradas no modelo $K A B$ (knowledge, attitudes, behaviour/conhecimento, atitude e comportamento). 0 modelo KAB, principal método para caracterizar a corrente individualista de educação em saúde, acredita, em relação linear, queo indivíduo, apósadquirir conhecimento, gera uma mudança de atitude e de comportamento, considerando que todas as pessoas agem demaneira lógica o tempo todo e reagem da mesma forma em qualquer situação, não levando em consideração as peculiaridades individuais².

Esseenfoquetradicional da educação em saúde, através da transmissão de conhecimentos, não dá importância aos processos de decodificação e ressignificação das informações recebidas pela população. As informações claras e objetivas não geram, por si, a reflexão, sensibilização e motivação para mudanças, pois essas são permeadas pelas representações sociais, pelos sentidos e pelos significados já existentes 3 .

Teixeira ${ }^{4}$ critica a educação em saúdetradicional, afirmando que o que ocorre é a educação bancária ${ }^{5,6}$, havendo a imposição de conceitos para os populares, esperando que eles passem a assumir o controle de sua saúde. Complementa afirmando que a população, como forma de resistência ou de acordo com as condições de vida, reinterpreta os termos técnicos-científicos restabelecendo a relação entre os cuidados e o corpo, pois a cultura biomédica baseia-se no funcionamento do organismo e, além do comprometimento fisiológico, o indivíduo tem as necessidades psicológicas e socioculturais.

A correnteestruturalista de educação em saúde enfoca a causação social do processo saúde-doença, em que as condiç̧̃̃es de vida e trabalho são fatores essenciais do adoecer humano. Influenciada, na década de setenta do século $X X$, por Paulo Freire, a corrente estruturalista ganha força, constituindo o método de educação popular em saúde ${ }^{2,5-7}$.
Assim, a educação popular em saúde foi buscada como principal alternativa para romper a tradição autoritária e normatizadora das práticas educativas em saúde. Falar de educação popular é, antes de tudo, falar de participação e inclusão social, pois a educação popular permite o desenvolvimento de diversas potencialidades da população ${ }^{1}$.

Paratanto, precisamos de profissionaisconscientes do seu papel de facilitador do processo ensino-aprendizagem que, superando seus preconceitos e o seu pensamento mecanicista, busquem a participação ativa dos educandos, utilizem metodologias apropriadas, priorizem a criação de espaços para o diálogo, valorizem e incorporem os saberes populares e almejem $0 \mathrm{em}$ powerment da população envolvida.

A categoria empowerment surge como núcleo filosófico central da promoção de saúde, pois é definida como um processo que permite aos indivíduos ecoletivos aumentarem o controlesobreos determinantes da saúde através da aprendizagem. Esta aprendizagem éfeita durante as diversas fases davida einclui o melhor enfrentamento diantedas doenças crônicas e das causas externas para possibilitar a conquista de uma melhor saúde ${ }^{8}$.

Assim, a utilização de metodologias participativas nas práticas educativas permite a tomada de consciência e reflexão de aspectos importantes na vida das pessoas, envolvendo emoções e sentimentos junto com as cognições, facilitando dessa forma a vivência de sentido, a emergência de novas percepções sobre fatos e acontecimentos e, sobretudo, a clarificação dos valores do grupo eo exercício do respeito à diferença, ou seja, ressignificar a experiência social eexercitar 0 respeito a si próprio e ao outro, num movimento dialógico de pensar e agir que permite queassumam e exercitem o papel de cidadãos ${ }^{3}$.

Partindo desse raciocínio, percebe-se que os portadores de doenças crônicas necessitam conhecer sua doença eapren der a conviver com ela, minimizando os efeitos causados através de uma adesão consciente ao tratamento ${ }^{9}$. Esta pesquisa foi desenvolvida com pacientes portadores de periodontites crônicas; a prevenção e controle dessa doença são dependentes da capacidade do paciente aderir ao tratamento, mantendo um acompanhamento profissional periódico.

Como também são sentidas as dificuldades do cirurgião-dentista em lidar com o paciente crônico, foram buscadas alternativas para esse problema.

Assim, temos como objetivo avaliar as oficinas de "aceitação ao paciente" para os cirurgiões- 
dentistas e de autocuidado para os portadores de periodontite crônica, analisando o aumento de conhecimentos sobre periodontite crônica, aspectos culturais do adoecer e enfocando a aceitação do paciente em relação à sua enfermidade.

\section{Métodos}

Para a utilização de referenciais pedagógicos que estimulem a participação ativa dos sujeitos investigados no contexto de ensino-aprendizagem e que estabeleçam um processo de educação coletiva, este estudo buscou trabalhar com metodologias ativas de aprendizagem ${ }^{1-3,5-7}$.

Inicialmente, foi realizado um estudo transversal descritivo, utilizando o referencial qualitativo através do estudo das concepções, crenças e atitudes dos pacientes portadores de periodontitecrônica. Não éobjetivo desteartigo a descrição dos resultados desta fase, que se encontram em outras publicações ${ }^{10,11}$, mas a contextualização, pois as atividades educativas propostas partiram desta fase da pesquisa.

A pós conclusão do diagnóstico inicial, foram verificadas diferenças nos modelos explicativo científico e popular; elevado conhecimento e motivação em relação à utilização de medidas preventivas; falhas na compreensão do caráter crônico das periodontites; geração de sentimentos de culpa, frustração e afastamento dos serviços de saúde ${ }^{10,11}$.

Os pesquisadores, junto com uma equipe de psicólogos com experiências em atividades grupais, a partir do diagnóstico, elaboraram uma proposta de atividade educativa, definiram os instrumentos de avaliação da interven ção e realizaram um teste piloto com um grupo de pacientes em dezembro de 2005.

As oficinas educativas, respeitando a cultura popular, foram realizadas no grupo-intervenção e dividida em três momentos:

(1) Apresentação dos participantes - solicitação dos motivos da procura pelo serviço. Indagação do que há em comum aos participantes, iniciando pelas explicações dosmembros do grupo. Em seguida, complementação da explicação da doença, através de desenhos. Por último, perguntava-se o que é uma doença crônica, apresentavam-se exemplos eforneciam-se explicações do caráter crônico das periodontites;

(2) Psicodrama didático - com cadeiras e a participação dos membros do grupo, fazia-se uma simulação do que ocorre na periodontite, usando-se como personagens o periodonto, 0 dente, as bactérias, a limpeza (escova e fio dental) e o tártaro, além da discussão dos seus fatores causais;

(3) Estresseeperiodontite-propunha-seque os participantes fechassem os olhos e realizavasea leitura de um texto sobreuma cena deestresse na préhistória. Após a leitura, solicitava-se que os membros do grupo descrevessem os seus fatores estressores do dia a dia emedidas utilizadas para combatêlos. Daí explicava-se a relação do estresse e periodontite e seincentivava a utilização de medidas para sua diminuição.

Os pacientes portadores de periodontite crônica em atendimento nas clínicas de periodontia do curso de odontologia da Universidade de Fortaleza (UNIFOR), da Universidade Federal do Ceará (UFC), da Universidade Camilo Castelo Branco (UNICASTELO) e da Associação Brasileira de Odontologia - secção Ceará (ABO-CE), no período de fevereiro a outubro de 2006 , foram convidados a participar desta fase da pesquisa.

A amostra foi uma casuística nos meses de estudo e a aleatoriedade foi garantida através da definição prévia de turnos semanais diferenciados entre o grupo de intervenção e de controle. Um turno de atendimento seria do grupo de interven ção e outro para o controle e assim sucessivamente. A amostra final constituiu-se de 51 pacientes participantes do grupo de intervenção e 47 do controle

Os critérios de inclusão foram: a idade entre 25 e 59 anos, presença de periodontite avançada e a disponibilidade pessoal dos pacientes.

A periodontite avançada foi avaliada através do índice PSR (Periodontal Screening and Recording), em que o paciente deveria ter no mínimo um sextante com periodontite avançada (PSR = 4). Os sextantes compreendem regiões das arcadas dentárias agrupadas entre os dentes 17-14, 13-23, 24-27, 37-34, 33-43 e44-47, em queo maior escore do PSR era registrado para cada um dos sextantes, que, se ausente, foi registrado com um $X$. Esse exame estava presente em todas as fichas clínicas.

No início e no final do tratamento, nos grupos de intervenção e controle, foram realizadas entrevistas estruturadas para avaliar o conhecimento em relação à periodontite crônica, utilizando um questionário, constando das seguintes variáveis: sexo, renda, idade, grau deescolaridade, dependência ao fumo, presença de diabetes, nome da doença, causas, fatores relacionados com o desenvolvimento da periodontite, intervenções realizadas quando a gengiva está inflamada, intervenções de autocuidado, procura 
pelo dentista, conhecimento sobre o caráter crônico. No questionário, havia uma pergunta aberta sobre a etiologia da doença, na qual o entrevistado poderia listar livremente essas causas enquanto que, no item sobre os fatores relacionados ao desenvolvimento da doença, os pacientes eram solicitados a dizer sim ou não, depois de uma pergunta estimulada. Houve um agrupamento de categorias afins e, por isso, nas tabelas, em alguns itens, há um número superior de respostas em relação aos entrevistados.

0 grupo-intervenção, além do tratamento clínico, entre o seu início e final, participou das oficinas educativas. 0 grupo-controle somente foi submetido ao plano de tratamento proposto pelaclínica. 0 plano detratamento clínico envolve não somente a interven ção direta na boca do paciente, mas instrução de higi ene oral, segundo os protocolos pré estabelecidos pelas clínicas de periodontia.

Foram realizadas oficinas de "aceitação ao paciente" com quatro turmas de especialização em periodontia, em Fortaleza (CE), com o intuito de sensibilizar os cirurgiões-dentistas para os aspectos emocionais, afetivos e culturais envolvidos nos portadores de periodontites crônicas.

As oficinas de "aceitação ao paciente" iniciavam-se com uma avaliação de um caso clínico em periodontia. A partir desta avaliação, era solicitado (em tarjetas) que os cirurgiões-dentistas refletissem os seus sentimentos envolvidos durante 0 atendimento de um caso semelhante. $0 \mathrm{~s}$ sentimentos afins eram agrupados. Em seguida, era feita uma exposição dialogada a partir das falas e sentimentos dos pacientes entrevistados na fase diagnóstica desta pesquisa. Encerrava-se a oficina com a sensibilização da necessidade do cuidado ao cuidador dos pacientes crônicos.

As participações eintervenções real izadas nos grupos de educação em saúde e nas oficinas de "acei tação ao paciente" foram registradas em um diário de campo.

\section{Análisedos dados}

Os dados foram elaborados através do programa Statistica (StatSoft, Inc.). As frequências observadas relativas aos momentos antes e depois nos grupos controle e intervenção foram analisadas pelo teste $\mathrm{McN}$ emar, com uma significância dep $<<0,05$.

O diário de campo foi sistematizado e as observações mais relevantes foram compiladas.

\section{Aspectos éticos}

As recusas e as solicitações de participar da pesquisa foram respeitadas. Os informantes tive ram suas identidades mantidas em sigilo ea concordância em participar da pesquisa foi firmada através da assinatura do termo de consentimento livre e esclarecido segundo a normatização da Resolução no 196/96 do Conselho Nacional de Saúde.

A pesquisa foi submetida à avaliação do Comitê de Ética em Pesquisa da UNIFOR, sendo aprovado em 26/07/2004 e todo o material coletado está sendo utilizado somente para fins de pesquisa.

\section{Resultados}

Tratava-se de uma amostra equitativa quanto ao sexo (48,98\% masculino e $51,02 \%$ feminino) . 0 grau de escolaridade $(55,1 \%$ até o primeiro grau completo) e a renda (54,08\% até dois saláriosmínimos) eram baixos. Quanto aos dois grupos considerados como de risco para periodontite crônica (diabéticos e fumantes), vimos quea adesão e/ou procura por serviços odontológicos especializados em periodontia é muito baixa entre os diabéticos (Tabela 1).

Em relação ao conhecimento sobre a periodontite crônica, houve pouca modificação no grupo-controle, após a realização dos procedimentos clínicos. Entretanto, existiu um incre mento do conhecimento em relação às causas, ao nome da doença (periodontite ou piorréia, como é popularmente conhecida) e aos fatores relacionados com o desenvolvimento da periodontite crônica no grupo de intervenção. Dentre os fatores causais, 0 aumento significativo ( $p<$ 0,05 ) ocorreu em relação à presença de bactérias específicas e ao fumo eà diminuição de pacientes que não sabiam da causa (Tabela 2).

Apesar de $39,8 \%$ de fumantes e ex-fumantes na amostra, o cigarro foi citado somente $13,7 \%$ e $8,1 \%$ nos momentos "depois", respectivamente nos grupos-controle eintervenção, como causador da doença, mas na pergunta estimulada sobre os fatores relacionados com a periodontite, a resposta foi positiva para ampla maioria dos entrevistados, antes e depois da intervenção, nos dois grupos, não gerando com isso significância estatística.

Os fatores populares agrupados na Tabela 2, no parâmetro "Relação com periodontite", foram falta de cálcio, estrutura dos ossos fraca (osteo- 
Tabela 1. Perfil da amostra do estudo, segundo sexo, idade, grau de instrução e renda. Fortaleza (CE), 2006.

\begin{tabular}{|c|c|c|}
\hline Parâmetro & $\mathrm{N}$ & $\%$ \\
\hline \multicolumn{3}{|l|}{ Sexo } \\
\hline M asculino & 48 & 48,98 \\
\hline Feminino & 50 & 51,02 \\
\hline \multicolumn{3}{|l|}{ Grau de instrução } \\
\hline Alfabetização & 34 & 34,69 \\
\hline Primeiro grau & 20 & 20,41 \\
\hline Segundo grau & 33 & 33,67 \\
\hline Superior & 11 & 11,22 \\
\hline \multicolumn{3}{|c|}{ Renda (salário mínimo) } \\
\hline$<1$ & 2 & 2,04 \\
\hline $1-2$ & 51 & 52,04 \\
\hline $2-5$ & 29 & 29,59 \\
\hline $5-10$ & 7 & 7,14 \\
\hline$>10$ & 0 & 0,0 \\
\hline Não informado & 9 & 9,18 \\
\hline \multicolumn{3}{|l|}{ Fumantes } \\
\hline Sim & 24 & 24,49 \\
\hline Não & 59 & 60,20 \\
\hline Ex-fumante & 15 & 15,31 \\
\hline \multicolumn{3}{|l|}{ Diabéticos } \\
\hline Sim & 4 & 4,08 \\
\hline Não & 94 & 95,92 \\
\hline
\end{tabular}

porose), excesso de antibiótico, café, dentes fracos para cárie dentária, comer alimentos quentes e bebidas al coólicas. As compreensões populares, presentes no parâmetro "Causa" (fatores relacionados à cárie) e no "Relação com a periodontite" (fatores populares), apesar de não possuírem comprovação científica, permanecem no imaginário popular, não diminuindo no grupo de interven ção e gerando significância estatística positiva $(p<0,05)$ no grupo-controle (Tabela 2$)$.

A Tabela 3 mostra o grau de conhecimento sobre as intervenções necessárias quando a gengiva está inflamada, o que fazer para cuidar e quando tratar. Nos momentos de agudização da doença, houve um aumento na necessidade de procura por dentista (nos dois grupos) e diminuição nas receitas populares (controle) eno uso de medicamentos antiinflamatórios e antibióticos (intervenção). No parâmetro "quando tratar", não houve significância estatística nos dois grupos. Como o enfoque da clínica é maior na técnica preventiva, na variável intervenção preventiva (escovação, uso do fio dental, bochechos com colutórios), não houve um incremento em nenhum dos grupos no parâmetro "gengiva inflamada" e"cuidar", apesar da elevada frequência antes e depois.

Tabela 2. Desempenho dos grupos de controle e intervenção, segundo conhecimento em relação à periodontite crônica. Fortaleza (CE), 2006.

\begin{tabular}{|c|c|c|c|c|c|c|}
\hline \multirow[b]{2}{*}{ Parâmetro } & \multicolumn{2}{|c|}{ Controle } & \multirow[b]{2}{*}{ Sig } & \multicolumn{2}{|c|}{ Intervenção } & \multirow[b]{2}{*}{ Sic } \\
\hline & Antes & Depois & & Antes & Depois & \\
\hline \multicolumn{7}{|l|}{ Causa } \\
\hline Má higiene & 23 & 32 & NS & 30 & 42 & NS \\
\hline Bactérias / infecção & 2 & 3 & NS & 0 & 13 & $*$ \\
\hline Fatores relacionados à cárie & 3 & 3 & NS & 5 & 6 & NS \\
\hline Doenças & 1 & 1 & NS & 0 & 6 & NS \\
\hline Fumo & 4 & 7 & NS & 0 & 7 & $*$ \\
\hline Padrão intrafamiliar & 0 & 0 & NS & 1 & 4 & NS \\
\hline Não sabe & 17 & 9 & NS & 12 & 0 & $*$ \\
\hline Outros & 4 & 3 & NS & 6 & 8 & NS \\
\hline \multicolumn{7}{|l|}{ Nome da doença } \\
\hline Gengivite & 10 & 12 & NS & 10 & 2 & $*$ \\
\hline Periodontite / piorréia & 8 & 9 & NS & 10 & 48 & $*$ \\
\hline Não sabe & 27 & 23 & NS & 28 & 1 & $*$ \\
\hline Outros & 2 & 3 & NS & 3 & 0 & NS \\
\hline \multicolumn{7}{|l|}{ Relação com periodontite } \\
\hline Má higiene & 87 & 93 & NS & 95 & 100 & NS \\
\hline Fatores populares & 125 & 166 & $*$ & 155 & 151 & NS \\
\hline Fumo & 37 & 45 & NS & 43 & 50 & NS \\
\hline Estresse & 18 & 25 & NS & 21 & 43 & $*$ \\
\hline Acesso a serviço espec. & 35 & 38 & NS & 45 & 48 & NS \\
\hline Fatores intrínsecos ao paciente & 52 & 58 & NS & 55 & 89 & $*$ \\
\hline
\end{tabular}

NS não significativo, $* P<0,05$. 
As receitas populares mencionadas na Tabela 3 foram bochechos com água morna e sal, vinagre, romã, bicarbonato, água oxigenada, aroeira e pedra-ume (alúmen de potássio). As receitas populares e o uso de medicamentos, através da automedicação, são medidas paliativas, que resolvem o problema por um curto intervalo de tempo. A pós as raspagens supra e subgengivais realizadas na clínica, a sensação de alívio é mais duradoura e, portanto, é sentida a importância do atendimento clínico.

0 foco da intervenção educativa foi a divisão de responsabilidades entre o paciente (necessidadedeuma boahigieneoral) eo serviço odontológico (necessidade de um bom tratamento e um programa de manutenção), pois foi compartiIhado o conhecimento quea periodontite possui, além dos determinantes locais relacionados à má higiene oral, os fatores intrínsecos do organismo (padrão intrafamiliar e baixa imunidade) e os socioambientais, como o estresse (Tabela 2). $\mathrm{Na}$ clínica, o enfoque é na má higiene oral, conhecimento senso comum para o paciente e de sua exclusiva responsabilidade, o que justifica a não significância estatística nos dois grupos, apesar da alta frequência no item "má escovação ou higiene" (Tabela 2) e a significância estatística ( $p$ < $0,05)$ presente no grupo de intervenção em relação ao estresse e fatores intrínsecos ao paciente
(Tabela 2) e no parâmetro "Cuidar" no item "intervenção clínica e preventiva" (Tabela 3).

A clínica está conseguindo manter a prevenção como um importantealiado do paciente, mas sem compreender o caráter crônico da doença, 0 que pode dificultar a manuten ção do autocuidado em saúde bucal e a adesão à terapia periodontal de suporte. 0 Gráfico 1 aponta que, no grupo-controle, não houve incremento na compreensão do caráter crônico da periodontite, ao contrário do grupo de intervenção.

A partir das anotações no diário de campo, observava-se nos pacientes um elevado agradecimento em relação ao conhecimento adquirido sobre a periodontite crônica ("meu doutor nunca me explicou direito o que eu tinha", "não sabia que não ia ficar boa", "não sabia nada disso") e a necessidade deum diálogo mais acentuado entre os profissionais e seus pacientes, que a cada sessão falavam cada vez mais de suas vidas, al egrias e tristezas.

Foi registrado um número elevado de pacientes com dificuldades psicoemocionais. Na me dida em que essas dificuldades eram compartiIhadas com a pesquisadora, procedia-se 0 encaminhamento para centros de referência em Fortaleza (CE), resultando num grande aprendizado etroca de vivências entre os participantes dos grupos.

Tabela 3. Desempenho dos grupos de controle e intervenção, segundo conhecimento em relação ao cuidado com pacientes portadores de periodontite crônica. Fortaleza (CE), 2006.

\begin{tabular}{|c|c|c|c|c|c|c|}
\hline \multirow[b]{2}{*}{ Parâmetro } & \multicolumn{2}{|c|}{ Controle } & \multirow[b]{2}{*}{ Sig } & \multicolumn{2}{|c|}{ Intervenção } & \multirow[b]{2}{*}{ Sic } \\
\hline & Antes & Depois & & Antes & Depois & \\
\hline \multicolumn{7}{|l|}{ Gengiva inflamada } \\
\hline Intervenção clínica & 5 & 28 & $*$ & 3 & 41 & $*$ \\
\hline Intervenção preventiva & 10 & 21 & NS & 15 & 14 & NS \\
\hline Receitas populares & 10 & 2 & $*$ & 3 & 0 & NS \\
\hline M edicamentos & 9 & 4 & NS & 12 & 1 & $*$ \\
\hline Não sabe & 17 & 3 & $*$ & 23 & 0 & $*$ \\
\hline \multicolumn{7}{|l|}{ Cuidar } \\
\hline Intervenção clínica & 7 & 4 & NS & 9 & 2 & NS \\
\hline Intervenção preventiva & 22 & 28 & NS & 25 & 19 & NS \\
\hline Clínica e preventiva & 5 & 11 & NS & 7 & 24 & $*$ \\
\hline Não sabe & 4 & 0 & NS & 3 & 0 & NS \\
\hline Outros & 10 & 7 & NS & 9 & 9 & NS \\
\hline \multicolumn{7}{|l|}{ Tratar } \\
\hline Antes de adoecer & 18 & 15 & NS & 23 & 31 & NS \\
\hline Primeiros sintomas & 25 & 29 & NS & 25 & 19 & NS \\
\hline N ão sabe & 4 & 3 & NS & 3 & 1 & NS \\
\hline
\end{tabular}

NS não significativo, $* P<0,05$. 


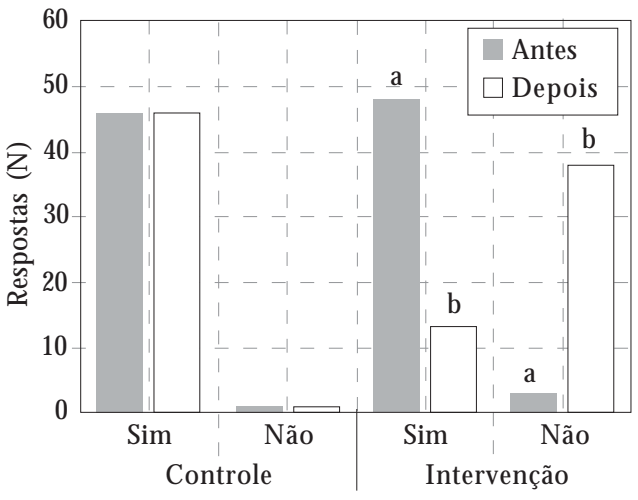

Gráfico 1. Desempenho dos grupos de controle e intervenção, segundo a compreensão que, ficarão curados após o tratamento clínico. Fortaleza (CE), 2006.

a, b $P<0,05$.

Nas oficinas de "aceitação ao paciente", os cirurgiões-dentistas relatavam suas dificuldades e inabilidades em motivar o paciente para a manutenção do autocuidado em saúde bucal ao longo da vida. Os temas recorrentes em todas as oficinas eram:

- Cansaço em atender o paciente com problemas recorrentes - palavras-chave utilizadas: impaciência, incompreensão, cansaço, desmotivação, tristeza, desanimo;

- Sentimentos negativos em decorrência da dificuldade de solução dos problemas - palavraschave utilizadas: desgosto, frustração, impotência, ineficácia, dificuldade, raiva, angústia;

- Somente um sentimento positivo que demonstrava a vontade de entender as dificuldades do paciente crônico: desafio.

\section{Discussão}

As doenças crônicas, na perspectiva das pessoas enfermas, trazem impactos no fluxo do cotidiano, necessitando reconduzir a vida para resolver impasses, decorrentes dos sintomas e incapacidades progressivas ${ }^{12}$.

Em alguns estudos na área da periodontia ${ }^{13}$, os pesquisadores buscam entender os aspectos envolvidos na aceitação dos pacientes em relação a sua doença. Uma série de recomendações é dirigida aos dentistas para melhorar a adesão: informação sobre a doença, simplificação das re- comendações, registro da aceitação, agendamento, reforço positivo, etc. Em última instância, a falha pela não aceitação da doença é somente do paciente e não se avalia e nem se questionam as medidas odontológicas utilizadas para a motivação do autocuidado.

Siqueira, Couto e Gusmão ${ }^{14}$, Toassi e Petry ${ }^{15}$ eSinkoç ${ }^{16}$ reforçam o modelo de muitos estudos que, intervindo nos aspectos biológicos do adoecimento, através de aulas demonstrativas e escovações supervisionadas, são suficientes para o desenvolvimento da motivação em saúde bucal, buscando convencer o paciente a fazer o que 0 profissional prescreve. 0 cuidado, entendido mais amplamente do que técnica de higiene bucal, não foi incorporado plenamente pela odontologia. Esse cuidado é essencialmente vocacionado pela enfermagem ${ }^{12,17}$.

A aceitação da periodontite crônica envolve não somente informação para o desenvolvimento da habilidade manual, mas a compreensão pelo cirurgião-dentista de toda a complexidade que envolve 0 acometimento de ser doente crônico ${ }^{12}$, compreen den do a diversidade eheterogeneidade da realidade social e a complexidade e singularidade da subjetividade humana, através do desenvolvimento da autonomia e da capacidade de superação das pessoas no enfrentamento das situações de saúde-doença, tanto a nível individual quanto coletivo ${ }^{1},{ }^{18}$.

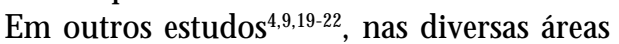
da saúde, têm-se desenvolvido atividades de educação esaúde com doentes crônicos, em que estes tornam-se sujeitos do processo de ensino-aprendizagem, envolvendo principalmenteconhecimento da doença, aceitação, motivação para 0 autocuidado, envolvimento familiar, investigação de variadas alternativas para a melhoria e/ou mudança nos hábitos e nas condições de vida com o objetivo defacilitar a convivência com suas doenças $^{19-21}$, necessitando, em alguns casos, inclusive, da participação de equipes multiprofissionais 9,22.

As concepções populares construídas culturalmente pela população não são fáceis de serem compreendidas pelos profissionais de saúde e vice-versa. E não ésomenteum momento clínico e/ou educativo que irá modificá-las. Por isso, a importância do desenvolvimento de atividades educativas problematizadoras nasáreas de abrangência das unidades de saúde, para uma maior aproximação e cumplicidade entre usuários e profissionais. Pois, a odontologia dialoga pouco com o saber popular, não avaliando a interação entre 0 que o profissional prescreve e a cultura do paciente. 
$N$ ations e Rebhun ${ }^{23}$ relatam que as atividades educativas não necessariamente precisam modificar as concepções populares das doenças, mas elas são importantes para abrir caminhos para a incorporação, no dia a dia dos usuários, demedidas preventivas que causem impactos nas prevalências das doenças.

A não significância estatística de quando tratar da periodontite crônica, preventivamente ou quando aparecem os principais sintomas, não é somenteuma inoperância das atividades clínicas e/ou educativas realizadas, mas uma resistência cultural ${ }^{24}$. A consciência preventiva, segundo Bosi e Afonso ${ }^{25}$, não deveria ser tão esperada pelos profissionais de saúde, já que esta postura decorre de uma experiência mediatizada pela teoria, o quenão corresponde à experiência existencial da maioria da nossa população.

No caso das doenças bucais, os estudos realizados ${ }^{26,27}$ confirmam que a dor e 0 incômodo são os únicos meios que possibilitam o autodiagnóstico de doente ou não e da necessidade de procura por uma assistência odontológica, reiterando o que Leriche, citado por Canguilhem ${ }^{28}$, relata quando afirma que a saúde é a vida no silêncio dos órgãos e a doença é aquilo que perturba os homens no exercício normal desuavida e em suas ocupações e, sobretudo, aquilo que os faz sofrer.

\section{Consideraçõesfinais}

Os aspectos culturais e psicológicos envolvidos no adoecer dos portadores de periodontite crônica são importantes de serem analisados para não gerarem uma desmotivação e descrença no processo terapêutico.

Assim, diante da pesquisa realizada, podemos concluir que houve uma maior compreensão dos processos patológicos dos pacientes que passaram por atividades de educação popular problematizadoras, do que os que participaram somente das orientações preventivas, durante 0 atendimento clínico, propostos pela educação em saúde tradicional, principalmente nos aspectos referentes ao conhecimento de qual doença pos- sui, suas causas e seu caráter crônico. Além disso, os pacientes possuem o conhecimento senso comum sobre a importância das técnicas pre ventivas e a má higiene oral como causadora da periodontite. Concluímos também que as compreensões populares diferem da odontologia científica, permanecendo no imaginário popular, independente das atividades clínicas e educativas realizadas.

Finalmente, é preciso identificar déficits de autocuidado e, durante 0 tratamento, desenvolver sistemas de compensação de acordo com a realidade sociocultural de cada paciente, sem culpabilidade, envolvendo necessariamente outros profissionais de saúde para lidar com a complexidade e singularidade do portador da periodontite como doença crônica, cujas características exigem uma dinâmica interativa entre profissionais interdisciplinares e paciente, modificando o modelo biomédico, que trabal ha entre bocas, dentes e línguas, para incorporar na alma da profissão o paradigma do cuidado ${ }^{29}$. O periodontista sozinho não é capaz de lidar com essa diversidade.

\section{Colaboradores}

SAS N uto trabalhou na concepção teórica, organização e execução das oficinas e entrevistas, elaboração, redação final do texto e revisão bibliográfica; SI F Amorim participou da organização e execução das oficinas e entrevistas; M K N ations trabalhou na concepção teórica, el aboração eredação final do texto; ICC Costa trabalhou na concepção teórica e redação final do texto. 


\section{Referências}

1. Vasconcelos EM. Educação popular e a atenção à saúde da família. 2a ed. São Paulo: Hucitec; 2001.

2. Abegg $C$. Notas sobre a educação em saúde bucal nos consultórios odontológicos, unidades de saúde e nas escolas. Ação Coletiva 1999; 2:25-28.

3. Silva C. M etodologias participativas para trabalhos de promoção de saúde e cidadania. São Paulo: Vetor; 2002.

4. Teixeira ER. Representações culturais de clientes diabéticos sobre saúde, doença e autocuidado. $R$ Enferm UERJ 1996; 4:163-169.

5. Freire P. Pedagogia do oprimido. 17 a ed. São Paulo: Paz e Terra; 1996.

6. Freire P. Pedagogia da esperança: um reencontro com a pedagogia do oprimido. 3a ed. São Paulo: Paz e Terra; 1994.

7. Vasconcelos EM. Educação popular nos serviços de saúde. 3a ed. São Paulo: Hucitec; 1997.

8. Carvalho SR. Os múltiplos sentidos da categoria "empowerment" no projeto de promoção à saúde. Cad Saude Publica 2004; 20:1088-1095.

9. Rabelo SL, Padilha MICS. Compreensão dos déficits de autocuidado a partir da prática assistencial. Texto Contexto Enferm 2000; 9:312-323.

10. N uto SAS, Costa ICC, N ations M K. M otivação do autocuidado na periodontite crônica: um estudo qualitativo. Periodontia 2006; 16:76-82.

11. Nuto SAS, Nations MK, Costa ICC. Aspectos culturais na compreensão da periodontite crônica: um estudo qualitativo. Cad Saude Publica 2007; 23:681690.

12. Lira GV, Nations MK, Catrib AM F. Cronicidade e cuidados de saúde : o que a antropologia da saúde tem a nos ensinar? Texto Contexto Enferm 2004, 13:147-155

13. Wilson Jr TG. Compliance: a review of the literature with possible applications to periodontics. J Pe riodontal 1987; 58:706-714

14. Siqueira JC, Couto GBL, Gusmão ES. Escovação no controle da placa: avaliação do ensino e motivação em escolares. RGO 2001; 49:127-132.

15. Toassi RFC, Petry PC. Motivação no controle do biofilme dental e sangramento gengival em escolares. Rev. Saude Publica 2002; 36:634-637.

16. Sinkoç CR. Educação em saúde bucal e a motivação do paciente. Rev Odontol Univ Santo Amaro 2001; 6:40-43.

17. Foster PC, Janssens NP. Dorothea E. Orem. In: George JB, organizador. Teorias de enfermagem: os fundamentos para a prática profissional. 3a ed. Porto Alegre: Editora Artes M édicas; 1993. p. 90-107.
18. Catrib AM F, Pordeus AMJ, Ataíde M BC, Albuquerque VLM, Vieira NFC. Promoção de saúde: saber fazer em construção. In: Barroso M GT, Vieira NFC, Varela ZM V, organizadores. Educação em saúde: no contexto da promoção humana. Fortaleza: Edições Demócrito Rocha; 2003. p. 31-37.

19. Freitas MC, Santana ME. Implementação da estratégia de ensino-aprendizagem à família de paciente crônico. Rev. Bras. Enferm. 2002; 55:146-150.

20. Hoga LAK. A influência da crença religiosa na motivação de uma cliente para o autocuidado. Rev Esc Enf USP 1993; 27:73-85.

21. Nunes AM P. M otivação para 0 autocuidado, um diagnóstico indispensável na assistência e orientação de diabéticos. Texto Contexto Enferm 1993; 2:53-66.

22. Jardim PCV, Sousa ALL, M onego ET. Atendimento multiprofissional ao paciente hipertenso. Medicina (Ribeirão Preto) 1996; 29:232-238.

23. Nations M K, Rebhun LA. Mystification of a simple solution: oral rehydration therapy in N ortheast Brazil. Soc Sci M ed 1988; 27:25-38.

24. Scott J. Domination and the arts of resistance: hidden transcripts. New Haven-London: Yale University Press; 1990.

25. Bosi M LM, Affonso KC. Cidadania, participação popular e saúde: com a palavra, os usuários da rede pública de serviços. Cad Saude Publica 1998; 14:355-365

26. Martins EM. Saúde bucal, uma necessidade socialmente construída: um estudo da experiência da Clínica Odontológica São Geraldo [dissertação]. Belo H orizonte (M G): Faculdade de Educação, Universidade Federal de Minas Gerais; 1993.

27. Nations M K, Nuto SAS. "Tooth worms", poverty tattoos and dental care conflicts in Northeast Brazil. Soc Sci M ed 2002; 54:229-244.

28. Canguilhem G. 0 normal e o patológico. 4a ed. Rio de Janeiro: Forense Universitária; 1995.

29. Boff L. Saber cuidar: ética do humano-compaixão pela terra. 4a ed. Petrópolis: Vozes; 1999.

Artigo apresentado em 29/05/2008

Aprovado em 15/01/2009

Versão final apresentada em 15/02/2009 